\title{
Possible migration front of gas-related fluid inferred from 3-D seismic data in the eastern Nankai trough
}

\author{
Hironori OTSUKA ${ }^{1}$, Sumito MORITA ${ }^{2}$, Manabu TANAHASHI ${ }^{2}$, Juichiro ASHI ${ }^{1}$ \\ and Sadao NAGAKUBO ${ }^{3}$ \\ ${ }^{1}$ Dept. of Natural Environmental Studies, the University of Tokyo \\ ${ }^{2}$ Geological Survey of Japan, National Institute of Advanced Industrial Science and Technology \\ ${ }^{3}$ Japan Oil, Gas and Metals National Corporation / Japan Drilling Co., Ltd
}

\begin{abstract}
High resolution 3D seismic survey, "Tokai-oki to Kumano-nada", was conducted for methane hydrate exploration in the eastern Nankai Trough by METI in 2002. Our study focuses on zigzag-shaped specific reflectors on BSR margins on the 3D data. We call the reflectors "Foldback Reflectors (FBRs)" in this study. From the edge of BSR, the 1st FBR generally extends down to lower formation below the BSR crossing sedimentary horizons. The following FBRs (often the 2nd, sometimes 3rd and those of higher order) extend down from the edge of the upper FBR forming accordion-like shape. The 1st FBR indicates normal polarity (antiphase of BSR), and the following FBRs change their polarities alternately. FBRs are mostly developed in the well-stratified formation but not in the area of frequent fractures and the area of major lateral lithological change. The estimated dip directions of each FBRs are probably controlled by the dip direction of crossing formation. FBR generally corresponds to lateral seismic facies boundary between BSR distribution area and outside of the BSR area. The formation beneath the BSR shows dimmed facies characterized by relatively low amplitude and lack of high frequency components in contrast to outside of the BSR area with normal facies. Seismic velocity analysis suggests that FBRs correspond to velocity boundaries, where the dimmed faceis below the BSR coinsides with relatively low velocity. The polarities of FBRs are also consistent with such velocity changes. Such dimmed facies with low velocity and low amplitude anomaly suggests effects of gas components in the formation water. The lowest FBR does not cross major unconformities, which often exhibit negative polarity suggesting fluid bearing strata. In this case, the lowest FBR with negative polarity merges to the negative reflection of the unconformity. In addition, high amplitude layers are sometimes recognized at foldbacks convex to the outside of the BSR area. These high amplitude layers probably having higher permeability are interpreted as conduits of gas-related fluid from the BSR distribution side to the outside of the BSR area. From these facts, FBR can be regarded as an important proxy indicating migration front of gas-related fluid.
\end{abstract}

\section{INTRODUCTION}

For decades, development of new energy resources is expected to replace traditional fossil fuels. Methane hydrate is one of the possible candidates for new energy resources, especially for Japan. On the other hand, explosive dissociation of methane hydrate is thought to have much effect on global climate ${ }^{1)}$. Thus, estimating potential storage of methane hydrate is important task for us. Accordingly, understanding of accumulation process of methane hydrate is significantly important. Accumulation of methane hydrate is thought to be closely related to the migration of the fluid including methane. Though fluid pathways are not fully understood, permeable strata, faults ${ }^{2)}$, and unconformities $^{3)}$ are strong candidates for major conduits of fluids. In order to understand actual condition of methane accumulation, high-definition three-dimensional geological information is needed. The Nankai Trough, the most well-investigated area in Japan, is the largest methane hydrate bearing area. Methane hydrate-related BSRs (Bottom Simulating Reflectors) are widely reported in the trench inner slope and the forearc basins based on multichannel seismic reflection surveys.

\section{DATA \& METHODOLOGY}

High resolution three-dimensional (3D) seismic survey, "Tokai-oki to Kumano-nada", was conducted for methane hydrate exploration in the eastern Nankai Trough by Ministry of Economy, Trade and Industry (METI) in 2002. We analyzed two data sets from the survey, called "Tokai-oki area" and "Daini-Atsumi knoll area".

In addition to interpretation of time-migrated seismic sections, we applied seismic attribute 
analysis including instantaneous amplitude, instantaneous phase, instantaneous frequency, and semblance. Horizon of formation or the other specific reflectors are picked on 3D seismic profiles and extracted as 3D pixel data. Additionally, strikes of each reflector were calculated from the 3D pixel data.

\section{RESULTS}

\section{(1) Mapping of FoldBack Reflector(s)}

Our study focuses on a series of accordion-shaped reflectors with horizontal axis of fold back. They are connected to the edge of BSRs and alternate their polarities at every fold back hinge. We call the reflectors "Foldback Reflectors (FBRs)" in this study.

The 1st FBR folds back from the edge of a BSR with reversed polarity (antiphase of BSR), crosses sedimentary reflectors below BSR and continues into the next fold back. The following FBRs (often the 2nd, sometimes 3rd and higher) extend down from the edge of the upper FBR forming vertical accordion shape. Although 3D seismic data exhibits various shapes of FBR possibly according to lithology and structure of formation, polarity of each FBR is laterally successive. Observing form the vertical cross-section parallel to the axis of fold back, the FBSs are almost parallel to the bedding plane of the surrounding formation. Another important character of FBRs is that it never crosses major unconformities into lower strata.

The formation beneath the BSR shows dimmed facies in contrast to the outside of the BSR area showing clear reflectors. We applied standard seismic attributes analysis to supplement our observation on the seismic profiles. The instantaneous amplitude and instantaneous frequency attributes indicate relatively low amplitude and lack of high frequency components corresponds to dimmed seismic facies. In other words, FBRs roughly correspond to the lateral boundary of two different seismic facies; dimmed facies and clear reflectors. Mostly, a horizon of formation consists of continuous reflector with no polarity changes across the each FBR. This observation suggests distribution of successive strata surrounding FBRs. In most cases, FBRs are recognized in tilted strata such as limb of anticline. In addition, high amplitude reflectors are sometimes recognized at foldbacks convex to the outside of the BSR area. The schematic diagram of FBR is shown in Figure 1.

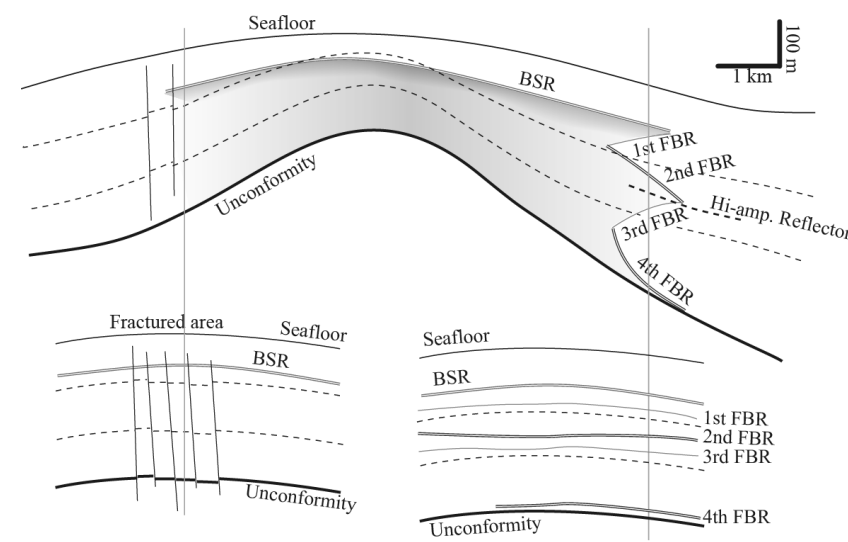

Figure 1 Schematic diagram of FBR (above). The two figures at bottom are the vertical cross sections of FBRs (right) and fractured area (left) respectively.

FBRs are observed in the area adjacent to the Tenryu Canyon and the Ryuyo Canyon ( $\mathrm{Ta}, \mathrm{Tb}$, and Tc area), the southeastern side of the Daiichi-Tenryu Knoll (Td area) and the area around the northern slope of the Daini-Atsumi Knoll (Aa nad $\mathrm{Ab}$ area). Distribution of FBRs and BSR are mapped on Figure 2. In the two areas, FBRs are mostly developed in the well-stratified formation but not in the area with pervasive fractures and major lateral lithological changes. FBRs also tend to be distributed around skirts of topographic high which constitute eastern margin of the outer ridge. The observed FBRs are clustered in northern slope of the outer ridge, whereas few FBRs are developed in the southern slope of the outer ridge with frequent compressive and strike-slip deformations related to major fault systems including the Kodaiba faults and the Tokai faults.

\section{(2) Horizon analysis}

Strikes of each FBR and the surrounding formation are calculated from picked 3D pixel data. Horizon of the surrounding formation was represented by the relatively continuous and clear reflector across the FBR. Strikes of FBRs and the surrounding formation are shown in Figure 3. The strike of the surrounding formation of the 1st FBR in the Td area (Td1) was substituted for that of the 2nd FBR (Td2) which was not be able to pick due to ambiguity of the horizon.

The histograms indicate FBR and the surrounding formation have totally similar strikes except the $\mathrm{Td}$ area. The mode value of each formation strike in the area of $\mathrm{Ta}, \mathrm{Tb}$, and $\mathrm{Tc}$ indicate homogeneously NE-SW direction corresponded to north-eastern slope of Daini-Tenryu Knoll. In these three areas, strikes of FBRs coincide with the surrounding formation having $\sim 23$ degrees deviation on an 
average. The histograms in the Daini-Atsumi Knoll area also show good correlation between strikes of FBR and stratum. The strike of formation indicates totally NE-SW direction in the Aa area, and E-W direction in $\mathrm{Ab}$ area. The averaged deviation of FBR strikes from formation strike in the Aa and the $\mathrm{Ab}$ areas shows 15 degrees. In contrast, the histograms in the Td area show large discrepancy between strikes of formations and FBRs; strike of the formation on $\mathrm{Td} 1$ indicates N-S direction, and that of the formation on $\mathrm{Td} 3$ indicates $\mathrm{E}-\mathrm{W}$ direction. The strikes of FBR indicate NW-SE direction on the 1st and 3rd FBRs, and NNE-SSW direction on the 2nd FBR though it has dispersed peaks. The deviation from direction of formation strike in the $\mathrm{Td}$ area shows maximum 60 degrees and 37 degrees on an average. In addition, the strikes of even numbered FBRs (reflectors which exhibit negative polarity) tend to exhibit distinct peaks in the histograms than odd numbered FBRs (reflectors of positive polarity) except the $\mathrm{Td}$ area.

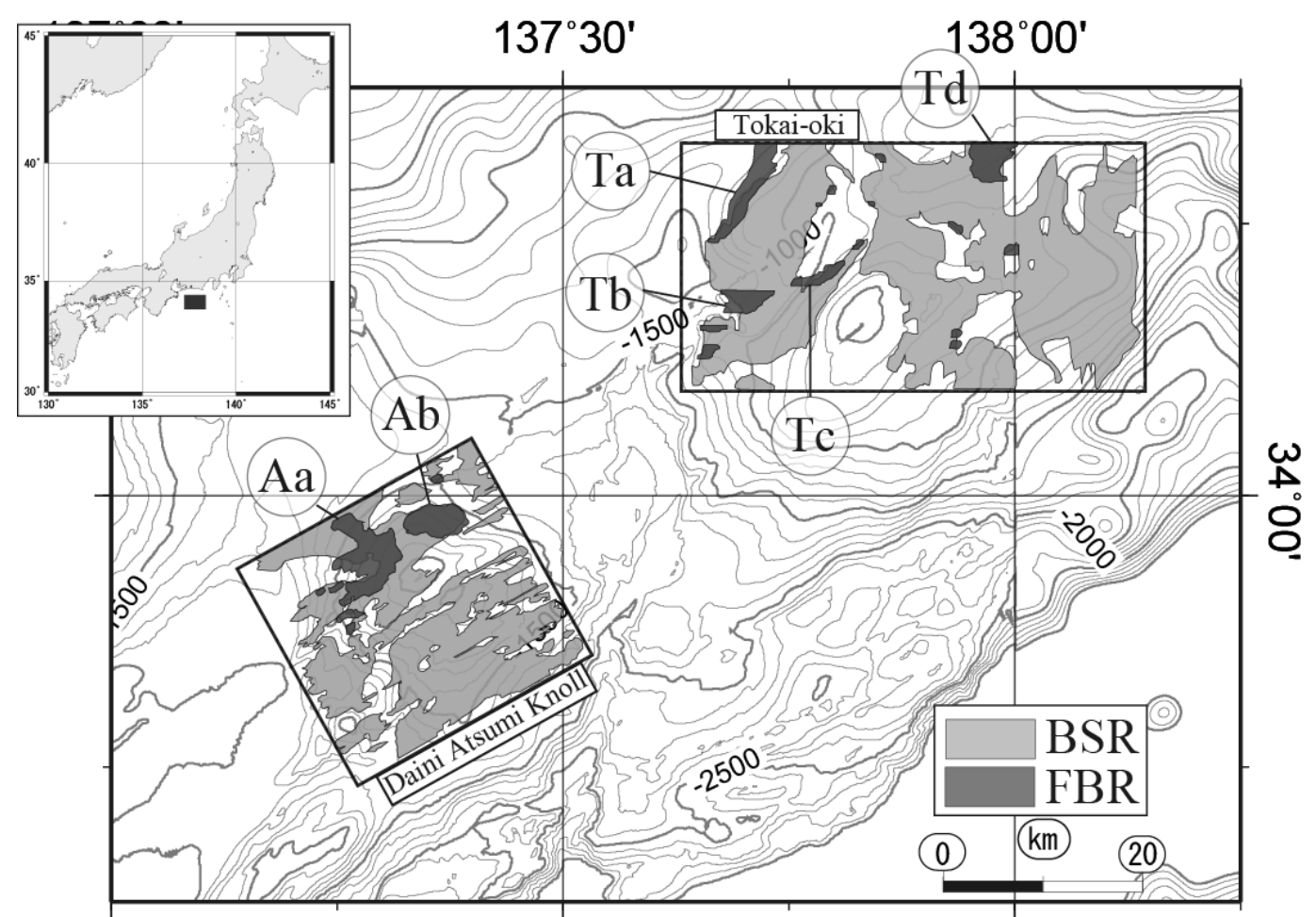

Figure 2 Location of 3D seismic surveys and distribution of FBR\&BSR.

\section{DISCUSSION}

It is inferred that FBR corresponds to lateral seismic facies boundary between BSR distribution area and outside of the BSR area. Seismic velocity analysis suggests that FBRs correspond to velocity boundaries, where the dimmed faceis below the BSR coinsides with relatively low velocity. The observation that polarities of FBRs alternately change with fold backs are also consistent with such velocity distribution. Such dimmed facies with low velocity and low amplitude anomaly suggests influences of gas components in the formation water.

The lowest FBR does not cross major unconformities, which often exhibit negative polarity suggesting fluid content. In this case, the lowest FBR with negative polarity merges with the negative reflection of the unconformity. Currently,
FBRs tend to be developed in tilted strata; especially in the observed area, in limbs of anticlines forming topographic high. Uplifting of the major three knolls in the study area commenced from Late Pleistocene with relatively high rate; maximum estimation of uplifting rate based on double-BSR observation is an order of $1 \mathrm{~cm} / \mathrm{yr}$ at Daiichi-Tenryu Knoll ${ }^{4}$. In the uplifting region, BSRs tend to exhibit stronger reflections in seismic profiles. Those stronger BSRs could be related to dissociation of gas hydrate due to drop in pore pressure ${ }^{5)}$. In addition, the dimmed seismic facies tend to be distributed below BSR developed in topographic high. Therefore, the regions developing FBRs can be regarded as the area where gas-related fluids tend to accumulate structurally. 


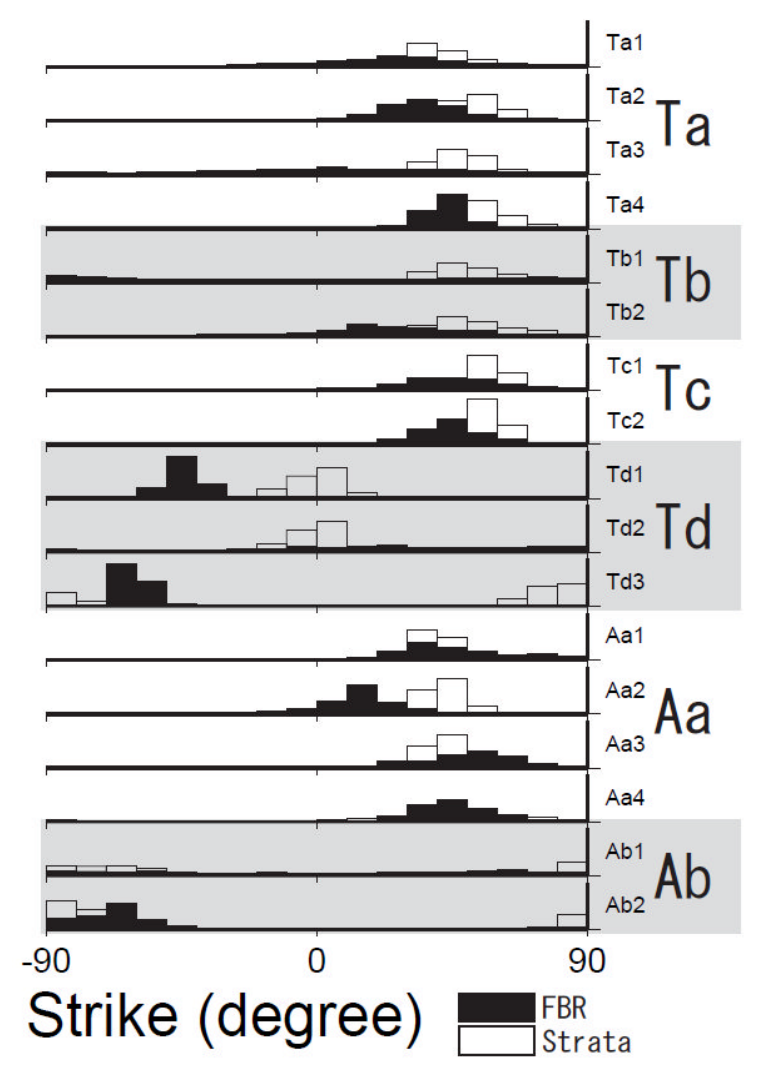

Figure 3 Histograms of calculated strikes of FBR (black) and formation (white). N-S direction is fixed at 0 degree. The suffix numbers after name of each area are order of foldbacks.

The results of strike plots suggest that dip direction of FBR is strongly controlled by dip of each surrounding formation. The result of horizon analysis and the distribution of dimmed seismic facies suggest formation process of FBR is primarily related to layer-parallel fluid migration. In the area of few (or no) FBRs which have frequent fractures and/or major lateral lithological changes, layer-parallel fluid migration could be prevented. The high amplitude layers recognized at foldbacks probably have higher permeability and are interpreted as conduits of gas-related fluid from the BSR distribution side to the outside of the BSR area. The feature that FBRs never cross major unconformities suggests that a series of layer-parallel fluid migration could be separated by those unconformities ${ }^{6}$.

The distinct strike mismatches between FBR and the surrounding formation in the $\mathrm{Td}$ area and the certain degree of deviation in the other areas imply the other factors controlling FBR shapes such as lateral lithological changes or distribution of faults. The appropriate interpretation for strike mismatches in the $\mathrm{Td}$ area is possibly lateral lithological changes because the 3D seismic profiles exhibit no vertical displacement of strata along the FBR, thus we suppose fault planes don't correspond to the shape of FBR. Possible interpretation for the dispersed plots of odd numbered FBRs on histogram is that they have irregular reflectors or relatively small dipping reflectors which are under the stronger influences of errors in the picked horizon data. The relatively small dipping reflectors might be related to gravity-controlled fluid migration. Consequently, the shape of FBR is possibly related to layer-parallel fluid migration and might be controlled by composite effects of formation architecture and lithology distributions.

\section{CONCLUSION}

This study revealed regularities of FBRs and mapped in the 3D seismic areas. FBRs extend down from the edge of BSR showing accordion shape and generally correspond to seismic facies and p-wave velocity boundaries. FBRs developed in suggestive region inclinable to accumulate gas-related fluids structurally, and are possibly related to layer-parallel fluid migration. From these facts, FBR can be regarded as an important proxy indicating distribution and migration of gas-related fluid.

ACKNOWLEDGMENT: This research used data obtained by Ministry of Economy, Trade and Industry (METI) and Japan Oil, Gas and Metals National Corporation (JOGMEC). This research is assigned as part of Research Consortium for Methane Hydrate Resources in Japan (MH21).

\section{REFERENCES}

1) Dickens, G. R., O’Neil, J. R., Rea, D. K., and Owen, R. M., 1995, Dissociation of oceanic methane hydrate as a cause of the carbon isotope excursion at the end of the Paleocene, Paleoceanography, 10(6), 965-972

2) Hustoft, S., Mienert, J., Bünz, S., and Nouzé, H., 2007, High-resolution 3D-seismic data indicate focused fluid migration pathways above polygonal fault systems of the mid-Norwegian margin, Marine Geology, 245, 89-106

3) Gay, A., Lopez, M., Berndt, C., and Séranne, M., 2007, Geological controls on focused fluid flow associated with seafloor seeps in the Lower Congo Basin, Marine Geology, 244, 68-92

4) Foucher, J.-P., Nouzé, H., and Henry, P., 2002, Observation and tentative interpretation of a double BSR on the Nankai slope, Marine Geology, 187, 161-175

5) Ashi, J. Tokuyama, H., and Taira, A., 2002, Distribution of methane hydrate BSRs and its implication for the prism growth in the Nankai Trough, Marine Geology, 187(1-2), 177-191

6) Hübscher, C. and Borowski, C., 2002, Seismic evidence for fluid escape from Mesozoic cuesta type topography in the Skagerrak, Marine Petroleum Geology, 23, 17-28 